\title{
Time-Domain Image Processing Using Dynamic Holography
}

\author{
Y. Ding, David D. Nolte, Michael R. Melloch, Senior Member, IEEE, and Andrew M. Weiner, Fellow, IEEE
}

\begin{abstract}
Coded ultrafast optical pulses can be treated as onedimensional (1-D) images in the time domain. We have converted space-domain images into time-domain images using diffraction from dynamic holograms inside a Fourier pulse shaper, with photorefractive quantum wells (QW's) used as the dynamic holographic medium. We present several examples, in which amplitude or phase modulation of the hologram writing beams modifies the complex spectrum of the femtosecond output, resulting in a time-domain image. Both storage and processing of time-domain images can be achieved, depending on the hologram writing geometry and power densities. Time-domain processing operations such as edge enhancement, Fourier transform, and correlation are demonstrated.
\end{abstract}

Index Terms-Holography, image processing, nonlinear optics, optical pulse shaping, optical signal processing, quantum-well devices, ultrafast optics.

\section{INTRODUCTION}

B Y ANALOGY with function generators and signal processors in electronics, the ability to arbitrarily manipulate ultrafast pulses is of great interest and practical importance in optical communication and information processing. Femtosecond (fs) lasers open possibilities to exploit the large bandwidth of optics (several terahertz $(\mathrm{THz})$ for commercially available sub-100-fs lasers). As one method to utilize this bandwidth, ultrafast pulses can be shaped and coded using Fourier synthesis techniques. Shaping of laser pulses on the femtosecond time scale was demonstrated in 1988 [1], in which the optical frequency components of a femtosecond pulse were manipulated by an amplitude or phase mask in the Fourier domain. By changing the mask, different shaped pulses were obtained. Extensive work has been done in this area [2] using different kind of masks such as photolithographic amplitude and phase masks [1], [3], dynamic liquid crystal spatial light modulators [4]-[7], accustooptic light modulators [8], computer-generated holographic plates [9], and dynamic holograms [10], [11]. Femtosecond pulse-shaping experiments using photorefractive quantum wells (PRQW's) [11] have demonstrated real-time control over the hologram with the material response time in the microsecond range.

Manuscript received October 1, 1997; revised February 24, 1998. This work was supported by the U.S. Department of Defense Focused Research Initiative through U.S. Air Force Office of Scientific Research under Grant F49620-951-0533, by the National Science Foundation under Grant 9708230 -ECS and by the Rome Laboratory under Grant F30602-96-2-0114.

Y. Ding and D. D. Nolte are with the Department of Physics, Purdue University, West Lafayette, IN 47907-1396 USA.

M. R. Melloch and A. M. Weiner are with the School of Electrical and Computer Engineering, Purdue University, West Lafayette, IN 47907 USA.

Publisher Item Identifier S 1077-260X(98)03771-X
Shaped pulses carrying information may have complicated temporal patterns which can be treated as one-dimensional (1-D) images in the time domain. The term "time-domain image" is most appropriate for cases when the shaped forms are obtained by converting space-domain images into the time domain. Time-space conversions are useful in femtosecond optical signal processing, optical transmultiplexing, image processing, and temporal pattern generation and recognition. Both time-to-space and space-to-time conversions have been demonstrated [12]-[16], and time-domain images have been obtained using both fixed and dynamic Fourier holograms [9], [17]. Processing time-domain images [17] (by analogy with processing conventional space-domain images) may open new possibilities for applications in optical communication, signal processing and data storage. Possible operations for time-domain image processing include: 1) single-image processing, such as noise suppression, defect correction, edge enhancement, time reversal, etc., where there is only one image involved, and 2) multi-image processing, such as convolution or correlation, where two or more images are involved. By using spectral holography [18], operations such as time reversal, correlation and matched filtering of time-domain femtosecond input optical pulses have been realized [19], [20]. Nonlinear spectral holography permits more elaborate signal-processing operations [21]. In this paper, we discuss in detail spaceto-time conversion using PRQW's for dynamic holography within a femtosecond pulse shaper and demonstrate the possibility of performing various processing operations, such as Fourier transform, edge enhancement, and correlation, as part of space-to-time conversion.

The second section of this paper explains the basic principle we used for time-domain image generation and processing, i.e., space-to-time conversion and processing during conversion, and describes the experimental setup, followed by a section demonstrating the suitability of PRQW for femtosecond timedomain image processing applications. The fourth and the fifth sections present results in the generation and processing of time-domain images, where we will give examples of single- and multi-image processing operations such as edge enhancement and correlation.

\section{PRINCIPLES AND EXPERIMENTAL SETUP}

Ultrafast optical pulses contain a wide bandwidth of, for example, $4 \mathrm{THz}$ for a typical 100-fs pulse duration with a Gaussian shape, corresponding to $10 \mathrm{~nm}$ at a center wavelength of $850 \mathrm{~nm}$ from a Ti:sapphire laser. Time-domain images can be generated and processed in the frequency domain using 


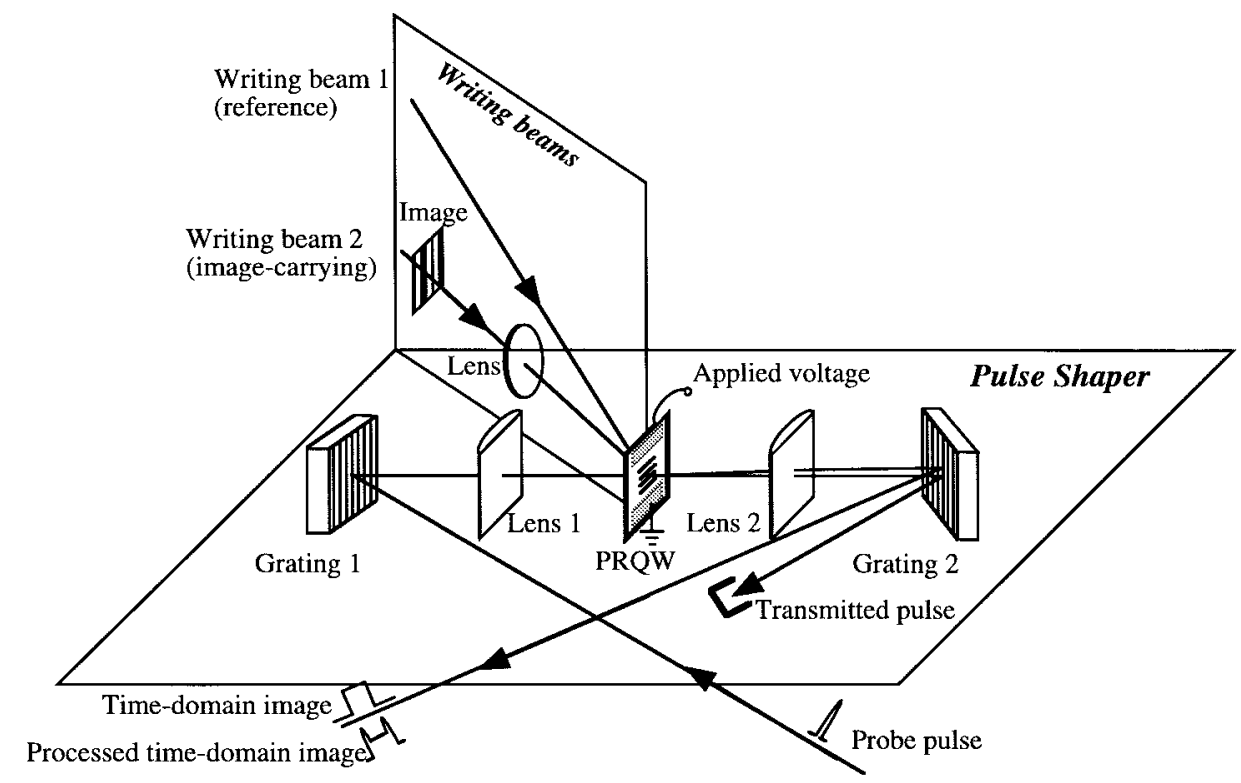

Fig. 1. Experimental setup for femtosecond time-domain image generation and processing using dynamic holograms written in photorefractive QW's, consisting two planes perpendicular to each other: pulse-shaper plane and hologram writing plane. The focusing length of the cylindrical lenses in the pulse shaper is $10 \mathrm{~cm}$ and the two gratings have a groove density of 1800 lines $/ \mathrm{mm}$, leading to a spatial spectrum separation of $0.35 \mathrm{~mm} / \mathrm{nm}$.

Fourier synthesis techniques. As an ultrafast optical pulse with a wide spectrum is dispersed by a dispersive element like a grating, its spectral components are spatially spread. If these components are filtered and modified by an amplitude or phase mask and then recombined, the resultant temporal output, which is the Fourier transform of the modified spectrum, contains the information of the mask. If the amplitude/phase mask is itself the Fourier transform of a certain space-domain image, for example a square aperture, the resultant temporal output envelope will take the same form as the space image, resulting in a time-domain image (a square wave). Any deviation of the mask from the Fourier transform of the space image will "distort" the converted time-domain image. Such deviation can be intentional and can be used to manipulate the time-domain images. The mask in the Fourier plane can be either a physical mask or a hologram. In our experiments, the mask is a dynamic hologram written in a PRQW by CW laser beams, which allows us to modify the output spectrum in situ. The temporal output is the Fourier transform of the frequency components diffracted from the hologram. By modifying the hologram writing beams, for example by projecting the Fourier transform of a space image onto the hologram medium by using a simple lens, time-domain images are generated by space-to-time conversion. The time-domain images can take different forms, if we control the parameters such as writing beam power densities, lens positions, etc.

The experimental setup is shown in Fig. 1 with main parameters in the figure caption. It is a dispersion-free $4 \mathrm{f}$ system allowing the synthesis of femtosecond pulses. The setup is similar to the conventional pulse-shaping apparatus [1] (pulse shaper) with two differences fitting the use of dynamic holography: first, a PRQW is placed in the Fourier plane in the pulse shaper instead of a mask, and second, the lenses used in the pulse shaper are cylindrical lenses instead of the conventional spherical lenses. The hologram is written in the
PRQW by two cw beams from either a diode laser operating at $685 \mathrm{~nm}$ or a He-Ne laser operating at $633 \mathrm{~nm}$. The power of each writing beam is $2-7 \mathrm{~mW}$. By using cylindrical lenses in the pulse shaper, the incident femtosecond beam at the Fourier plane remains unfocused in the vertical direction, so that its power density will be not so high as to erase the hologram, leading to optimal photorefractive diffraction. The Fourier transforms of the femtosecond probe beam and the diffracted beam by the cylindrical lenses are, therefore, 1-D transforms in the horizontal direction.

The femtosecond source is a mode-locked Ti-sapphire laser with a nominal pulsewidth of $100 \mathrm{fs}$ and a central wavelength adjusted to the excitonic absorption line of the QW's. The laser pulses are characterized to be transform-limited and have a Gaussian shape by interferometric second-harmonic generation (SHG) autocorrelation, power spectroscopy, and spectral interferometry. The femtosecond probe beam with a power of $2.6 \mathrm{~mW}$ and a diameter of $1.8 \mathrm{~mm}$ is incident on the first grating of the pulse shaper, where it is spectrally dispersed. The frequency components are diffracted from the hologram in the PRQW at the Fourier plane. The diffracted beam travels in the vertical plane determined by the two writing beams. The diffracted optical components are recombined by the second cylindrical lens and by the grating, forming the femtosecond output carrying the information of the hologram. The spectrum of the femtosecond output is captured through a spectrometer with a CCD array, and the temporal shape of the pulse is measured by electric-field cross correlation with an unshaped transform-limited reference pulse. Electric-field cross correlation is a technique suitable for weak ultrashort pulse characterization [22], [23], where the field-interferogram of the signal and reference pulses is recorded by scanning the time delay between the two pulses. For space-to-time conversion or single-image processing, a lens is put into one of the writing beams, called the image-carrying beam, to project 
a mask or its Fourier transform onto the PRQW, and the reference beam is unaltered. In the experiments of multi-image processing, both writing beams contain a lens. The image Fourier plane is $22^{\circ}$ off the Fourier plane of the pulse shaper, for technical simplicity. This has no detrimental effects on our results because of the long focal length of the lenses in the writing beams (100-200 mm). Neutral density filters are used to adjust the power densities of the two writing beams.

The pulse shaper is carefully aligned to be dispersion free with no PRQW in it, giving identical output pulses to the incident ones. Then a GaAs- $\mathrm{Al}_{x} \mathrm{Ga}_{1-x} \mathrm{As}$ PRQW is placed in the Fourier plane. Two samples were used in our experiments, one with $x=0.1$ and the other with 0.3 . The heavy-hole excitonic absorptions are centered at 847 and 836 $\mathrm{nm}$, respectively. Titanium-gold contacts were evaporated on the samples with a $1 \times 10 \mathrm{~mm}^{2}$ aperture. Across the $1-\mathrm{mm}$ gap, a field of $3-7 \mathrm{kV} / \mathrm{cm}$ is applied in the plane of the QW's, perpendicular to the growth direction (transverse field geometry [24], [25]). The angle between the two writing beams is 3.3 degrees and the output diffraction efficiency is on the order of $0.1 \%$.

\section{DifFRACTION OF FEMTOSECOND PULSES FROM PRQW's IN THE TRANSVERSE-FIELD GEOMETRY}

As a dynamic holographic medium, PRQW's have high nonlinear sensitivities (refractive index change $\Delta n \sim 10^{-2}$ ) with small driving power densities $\left(\sim 10 \mu \mathrm{W} / \mathrm{cm}^{2}\right)$ and fast response times ( $\sim 10 \mu \mathrm{s})$ [24], [25], [26]). The holograms in such materials are thin and the diffraction is in the Raman-Nath regime with no Bragg-matching necessary, providing much larger space-bandwidth products than bulk materials [27], which is important for Fourier filtering in image processing applications. PRQW's can also be designed using semiconductor bandgap engineering to match different application wavelengths, such as $1.55 \mu \mathrm{m}$ for optical fiber communications [28].

For dynamic Fourier manipulation and processing of timedomain images, an ideal holographic material should have a flat amplitude and phase response over the bandwidth of the pulses. The diffraction from the hologram in such a material would not distort the probe pulse shape if the hologram itself is not modified by any masks. Photorefractive holograms in PRQW are based on the electroabsorption (electric-field-induced absorption) and electrorefraction (electric-field-induced refractive index change) of quantumconfined excitons. This effect has a typical bandwidth of $5 \mathrm{~nm}$ [29], which limits the 10-nm bandwidth for a typical 100-fs Gaussian pulse at $850 \mathrm{~nm}$ and broadens the diffracted pulses by a factor of two. Most importantly, the effect by itself is strongly wavelength dependent. The oscillatory behavior as a function of wavelength might be expected to lead to severe distortion of the diffracted pulse shape. However, it was experimentally observed that both spectrum and temporal shape of the diffracted pulse appear to be nearly Gaussian with a time-bandwidth product of 0.43 , which is very close the that for Gaussian pulses (0.44), although the limited bandwidth of the quantum-confined excitonic transitions broadens the diffracted pulses [29]. This implies that the diffracted pulse is

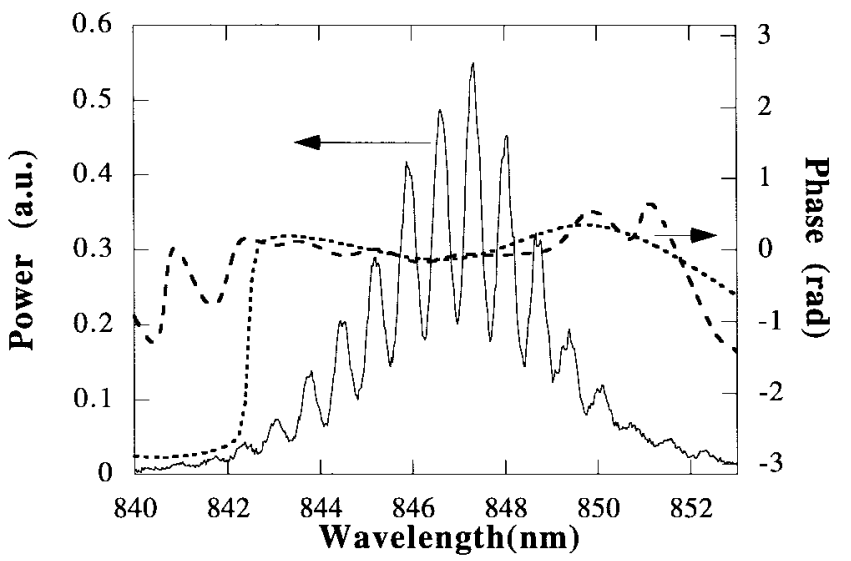

Fig. 2. Phase of the diffracted pulse from a 10\% Al-barrier PRQW in the pulse shaper. The dashed line represents the phase extracted from the spectral interferometry pattern (solid line) between the reference pulse and diffracted pulse and the dot line is the phase calculated from the electroabsorption and electrorefraction data.

nearly transform-limited and the phase of the diffracted pulse in the frequency domain is linear.

To understand the linear phase, the field amplitude of the first-order diffraction of a femtosecond incident pulse $E_{\mathrm{IN}}(\omega)$ from a photorefractive grating in PRQW is given by [29]

$$
E_{D}(\omega)=E_{\mathrm{IN}}(\omega) F_{T}(\omega) F_{D}(\omega)
$$

with the transmission function

$$
F_{T}(\omega)=\exp [i \tilde{n}(\omega) k L]=\exp (-\alpha(\omega) L / 2+i n(\omega) k L)
$$

and the diffraction function

$$
F_{D}(\omega)=\frac{1}{2}(i \Delta n(\omega) k L-\Delta \alpha(\omega) L / 2)
$$

where $\tilde{n}$ is the complex refractive index, $L$ is the interaction length and $k$ is the wavevector in vacuum. As long as the frequency dependence of the background absorption is not strong, which is the case when the excitonic absorption line is broadened by the applied electric field in the transverse-field geometry, where the electric field is parallel to the QW layers, the phase of the diffracted field $E_{D}(\omega)$ is mainly determined by the phase of the diffraction function $F_{D}(\omega)$ in (3)

$$
\Phi(\omega)=-\arctan \left(\frac{2 k \Delta n(\omega)}{\Delta \alpha(\omega)}\right)
$$

indicating that the phase is given by the ratio of the electrorefraction $\Delta n(\omega)$ and the electroabsorption $\Delta \alpha(\omega)$, which are related by the Kramers-Kronig relation [30]

$$
\begin{aligned}
& \Delta n(\omega)=\frac{c}{\pi} P \int_{0}^{\infty} \frac{\Delta \alpha\left(\omega^{\prime}\right)}{\omega^{\prime 2}-\omega^{2}} d \omega^{\prime} \\
& \Delta \alpha(\omega)=\frac{4 \omega^{2}}{c \pi} P \int_{0}^{\infty} \frac{\Delta n\left(\omega^{\prime}\right)}{\omega^{2}-\omega^{\prime 2}} d \omega^{\prime}
\end{aligned}
$$

where $P$ stands for the Cauchy principal value and $c$ is the light velocity. Using the electroabsorption measurement $\Delta \alpha(\omega)$ and the calculated electrorefraction data $\Delta n(\omega)$ and (4), we calculate the phase of the diffracted beam in the frequency domain as shown in Fig. 2. The phase of the diffracted pulse can be measured experimentally using spectral interferometry 
[31], which is a versatile linear technique for determining both phase and shape of weak femtosecond pulses, where the spectrum of a reference pulse interferes with the spectrum of the diffracted pulse with a chosen time delay. Fig. 2 shows the spectral interferometry pattern with a time delay of $1500 \mathrm{fs}$ and the phase of the diffracted pulse extracted from it, together with the phase calculated from (4) using electroabsorption and electrorefraction data. After subtracting a linear part of the curves, the phase is quite flat around the center wavelength of $847 \mathrm{~nm}$ for the PRQW sample used.

The linear phase in the diffracted pulse is a result of the character of the transverse-field PRQW devices, where electroabsorption is caused by the broadening of the transition lineshape. The characteristic electroabsorption spectrum $\Delta \alpha(\omega)$ is approximately the second derivative of a Gaussian transition lineshape [24], which is an even function. The electrorefraction $\Delta n(\omega)$ is related to $\Delta \alpha(\omega)$ by the Kramers-Kronig relation, which transforms an even function to an odd one. Therefore, to first order the phase $\Phi(\omega)$ is linear in frequency.

We assume an excitonic absorption line that is Gaussian at room temperature [32]

$$
\alpha(\omega)=\sqrt{\pi \ln 2} \frac{\hbar \omega_{p}^{2}}{c n} \frac{f}{\Gamma} \exp \left(-4 \ln 2 \omega^{2} / \Gamma^{2}\right)
$$

where $\Gamma$ is the FWHM of the lineshape, $h=2 \pi \hbar$ is the Planck constant, $\omega_{p}$ is the ionic plasma frequency, and $f$ is the oscillator strength.

For the transverse-field geometry at relatively small fields (typically $<15 \mathrm{kV} / \mathrm{cm}$ ), the linewidth becomes $\Gamma=\Gamma_{0}+\Delta \Gamma$ with the linewidth without field $\Gamma_{0}$. The electroabsorption is then

$$
\begin{aligned}
\Delta \alpha(\omega)= & \frac{d \alpha(\omega)}{d \Gamma} \Delta \Gamma \\
= & \sqrt{\pi \ln 2} \frac{\hbar \omega_{p}^{2}}{c n} \frac{f}{\Gamma^{2}}\left(-1+\frac{8 \ln 2}{\Gamma^{2}} \omega^{2}\right) \\
& \times \Delta \Gamma \exp \left(-4 \ln 2 \omega^{2} / \Gamma^{2}\right) .
\end{aligned}
$$

The maximum of the absolute electroabsorption $|\Delta \alpha(\omega)|$ is at the center frequency, i.e., $\omega=0$ with the value of

$$
\Delta \alpha_{\max }=\sqrt{\pi \ln 2} \frac{\hbar \omega_{p}^{2}}{c n} \frac{f}{\Gamma^{2}} \Delta \Gamma .
$$

Near the central frequency of the excitonic absorption, $k$ is approximately a constant because the linewidth $\Gamma$ is much smaller than the central frequency, and (7) can be written as

$$
\begin{aligned}
\Delta \alpha(\omega) \approx & -\sqrt{\pi \ln 2} \frac{\hbar \omega_{p}^{2}}{c n} \frac{f}{\Gamma^{2}} \cos \left(\frac{4 \sqrt{\ln 2}}{\Gamma} \omega\right) \\
& \times \Delta \Gamma \exp \left(-4 \ln 2 \omega^{2} / \Gamma^{2}\right) .
\end{aligned}
$$

Using $\exp \left(-x^{2}\right) \approx 1-x^{2}$, it can be further rewritten as

$$
\begin{gathered}
\Delta \alpha(\omega) \approx-\frac{1}{2} \sqrt{\pi \ln 2} \frac{\hbar \omega_{p}^{2}}{c n} \frac{f}{\Gamma^{2}} \Delta \Gamma\left[\cos \left(\frac{(4+2 \sqrt{2}) \sqrt{\ln 2}}{\Gamma} \omega\right)\right. \\
\left.+\cos \left(\frac{(4-2 \sqrt{2}) \sqrt{\ln 2}}{\Gamma} \omega\right)\right]
\end{gathered}
$$

where we neglected the $(\omega / \Gamma)^{4}$ - and higher-order terms.
The Kramers-Kronig relation transforms the cosine functions to the - sine functions. Therefore, the electrorefraction from (5) and (10) is

$$
\begin{aligned}
\Delta n(\omega) & \approx \frac{1}{4 k} \sqrt{\pi \ln 2} \frac{\hbar \omega_{p}^{2}}{c n} \frac{f}{\Gamma^{2}} \Delta \Gamma \\
& \cdot\left[\sin \left(\frac{(4+2 \sqrt{2}) \sqrt{\ln 2}}{\Gamma} \omega\right) \sin \left(\frac{(4-2 \sqrt{2}) \sqrt{\ln 2}}{\Gamma} \omega\right)\right]
\end{aligned}
$$

After some algebra, it can be written as

$$
\begin{aligned}
\Delta n(\omega) \approx & \frac{1}{2 k} \sqrt{\pi \ln 2} \frac{\hbar \omega_{p}^{2}}{c n} \frac{f}{\Gamma^{2}} \sin \left(\frac{4 \sqrt{\ln 2}}{\Gamma} \omega\right) \\
& \times \Delta \Gamma \exp \left(-4 \ln 2 \omega^{2} / \Gamma^{2}\right) .
\end{aligned}
$$

From (9) and (12), the phase of the diffraction function $F_{D}(\omega)$ in (3) is then

$$
\Phi(\omega)=-\arctan \left(\frac{2 k \Delta n(\omega)}{\Delta \alpha(\omega)}\right)=\frac{4 \sqrt{\ln 2}}{\Gamma} \omega
$$

which is a linear function with $\omega$. A linear phase in the frequency domain introduces only a delay of the output pulse in the time domain but not distortion. The slope in (13) is inversely proportional to the linewidth and is independent of the applied field.

The amplitude of the diffraction function can also be deduced from (9) and (12), which is proportional to $\left[(\Delta n k L)^{2}+\right.$ $\left.(\Delta \alpha L / 2)^{2}\right]^{\frac{1}{2}}$. It is easily seen that $\left|F_{D}(\omega)\right|^{2}$ takes the same Gaussian shape as the absorption, which determines the bandwidth limit of the PRQW. Therefore, the output diffracted pulse is bandwidth-limited with a Gaussian shape, as experimentally observed. This distortionless quality makes the PRQW's suitable for use in pulse manipulation.

\section{Generation of Time-Domain IMAGES BY SPACE-TO-TIME CONVERSION}

In this section, we discuss the generation of time-domain images by space-to-time conversion using holographic diffraction from a PRQW, where a conventional image in the spacedomain is converted dynamically into the time domain. Using the experimental setup in Fig. 1, we realize the space-totime conversion by projecting the Fourier transform of a conventional image onto the PRQW in the pulse shaper. If the hologram amplitude in the PRQW is proportional to the Fourier-transformed image, the shaped temporal pattern, which is the Fourier transform of the diffracted spectrum from the hologram, should take on the same shape as the input spacedomain image.

The interference pattern of the two coherent writing beams has a modulation depth of

$$
\begin{aligned}
m(x) & =\frac{2\left|E_{1}^{*} E_{2}(x)\right|}{\left|E_{1}\right|^{2}+\left|E_{2}(x)\right|^{2}+\delta\left|E_{\text {Probe }}\right|^{2}} \\
& =\frac{2 \sqrt{I_{1} I_{2}(x)}}{I_{1}+I_{2}(x)+\delta I_{\text {Probe }}}
\end{aligned}
$$


with field amplitude $E_{1}$ (power density $I_{1}$ ) of the reference writing beam (spatially homogenous), those of the imagecarrying beam $E_{2}(x), I_{2}(x)$, and of the femtosecond probe beam $E_{\text {Probe, }} I_{\text {Probe }}$, respectively. $\delta$ is a factor describing the ratio of the photocarriers induced at the probe and writing wavelengths. Because the femtosecond probe beam has a much longer wavelength $(850 \mathrm{~nm})$ than that of the writing beams (633 or $685 \mathrm{~nm}$ ), it was shown that the erasure of the grating by the probe beam is much weaker than the effect of the writing beams [33] (i.e., $\delta \ll 1$ ) due to the difference in the photoconductivity and interaction length at the two wavelengths. From (14), $m$ depends only on the power density ratio of the two beams $I_{2} / I_{1}$ and has the maximum value of unity when $I_{2}=I_{1}$ (neglecting erasure). The hologram written in the PRQW is proportional to $m$ as long as $m$ is small. As $m$ approaches unity, the amplitude of the photorefractive grating, and therefore the diffracted electric field amplitude as well, become sublinear with $m$ [34], [35]. In holographic recording, the hologram pattern is a faithful replica of the original electric field distribution only if the power density of the image-carrying beam is much less than the power density of the reference beam and the material response is linear in power density. In this case $\left(I_{1} \gg I_{2}(x)\right.$ and $I_{1}$ does not depend on $x), m(x) \sim E_{2}(x)$, and the hologram has the same distribution as the image-carrying beam $E_{2}(x)$.

As an example, a single slit with a width of $380 \mu \mathrm{m}$ is used as a space-domain image and is put into the image-carrying beam. This image is Fourier transformed onto the PRQW by a cylindrical lens with a focal length of $f=150 \mathrm{~mm}$, performing the Fourier transform one-dimensionally. The power density distribution at the Fourier plane takes the form of a squared sinc function when $x / f \ll 1$

$$
\left|E_{2}(x)\right|^{2}=I_{2 \max } \operatorname{sinc}^{2}\left(\frac{2 \pi D x}{\lambda f}\right)=I_{2 \max }\left(\frac{\sin \left(\frac{2 \pi D x}{\lambda f}\right)}{\frac{2 \pi D x}{\lambda f}}\right)^{2}
$$

for a slit width $2 D$, writing wavelength $\lambda$, and the coordinate $x$ in the Fourier plane of the Fourier lens. Using $2 D=380$ $\mu \mathrm{m}, f=150 \mathrm{~mm}$, and $\lambda=685 \mathrm{~nm}$, the FWHM of the main maximum of the distribution in (15) is approximately $250 \mu \mathrm{m}$. The power density of this maximum is, therefore, not more than twice as large as the power density before the Fourier imaging, which is approximately equal to the reference power density. Neutral density filters are therefore used to reduce the image-carrying beam power density by an order of magnitude to ensure that the reference beam has higher power density than the image-carrying beam everywhere. The measured diffracted power spectrum from the hologram also takes the sinc squared distribution as shown in Fig. 3(a). For comparison, the theoretical curve using (14) and (15) is also given, where the coordinate $x$ is translated into wavelength using the spectral dispersion of the pulse shaper of 0.35 $\mathrm{mm} / \mathrm{nm}$. The PRQW spectral response with a power density full-width at half-maximum (FWHM) of $4.2 \mathrm{~nm}$ for the $30 \%$ Al-barrier sample is also included. The spectrum agrees well with the simple theory. The finite spectral resolution of both the pulse shaper and the measurement apparatus, and the angle

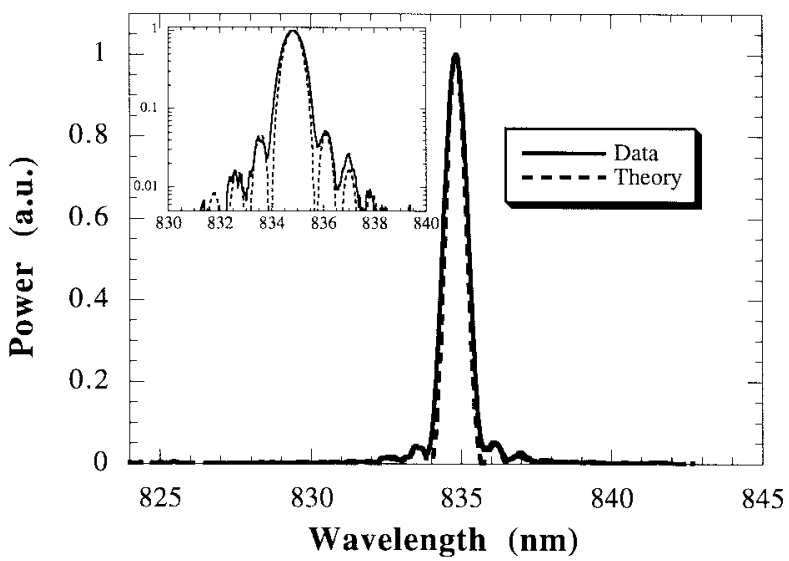

(a)

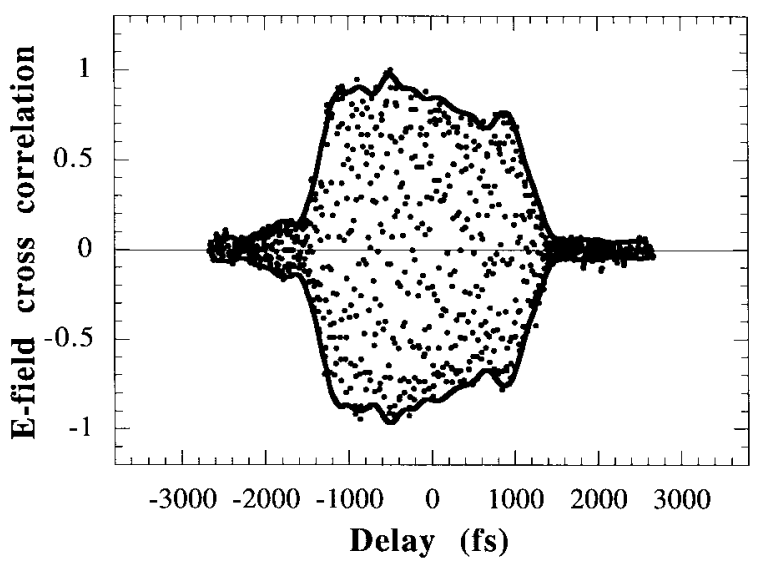

(b)

Fig. 3. (a) Spectrum and (b) the electric-field cross-correlation data and the extracted envelope of the diffracted temporal image when the Fourier transform of a single slit is projected onto the PRQW. The reference beam power density is higher than the image-carrying beam power density. This shows the space-to-time conversion. The corresponding theoretical calculation of the spectrum at $I_{1} / I_{2 \mathrm{Max}}=10$ is also given in (a).

between the image Fourier plane and the Fourier plane in the pulse shaper cause the minima of the power density distribution in the experimental data to remain finite. The temporal shape of the diffracted (or reconstructed) femtosecond outputs is measured by electric-field cross correlation as shown in Fig. 3(b), which corresponds to the input space-domain image; a square-wave packet. It is important to note that Fig. 3(b) contains the electric-field cross-correlation data. The side lobes would appear much smaller if the power density rather than the field amplitude were plotted.

\section{Processing of Femtosecond Time-Domain Images}

The images can be manipulated as they are converted from the space-domain to the time domain by changing the hologram writing power density, writing geometries and the relation between the input images, etc. Single image processes as well as multiple image processes can be realized.

\section{A. Single-Image Processing: Edge Enhancement, Fourier Transform, Noise Suppression, and Time Reversal}

Many applications require images with enhanced features, such as edges and noise, or certain transformations of a image, 


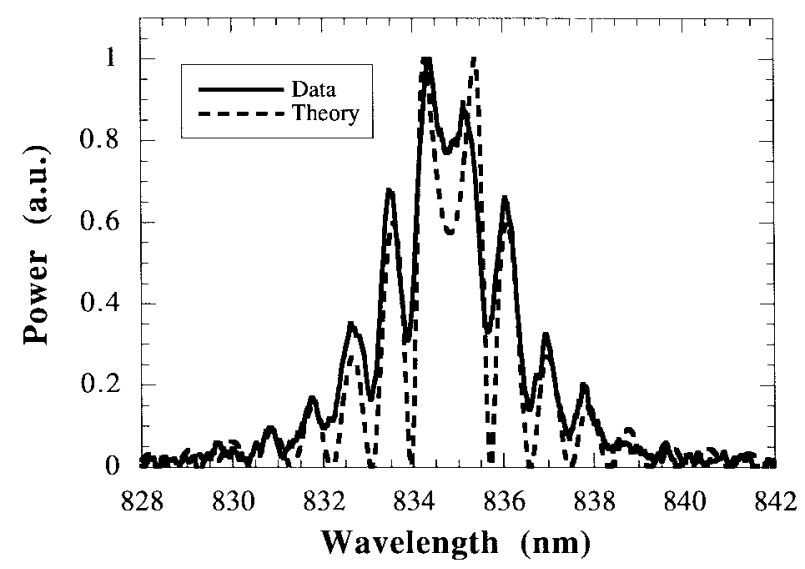

(a)

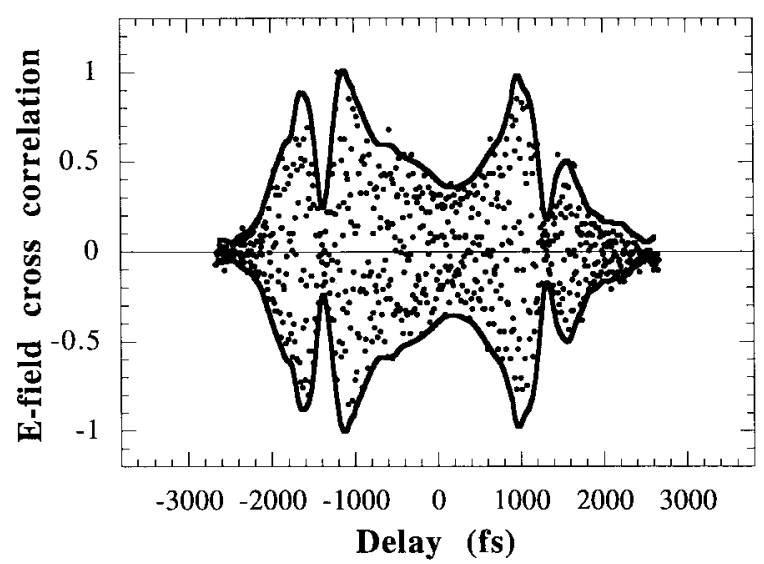

(b)

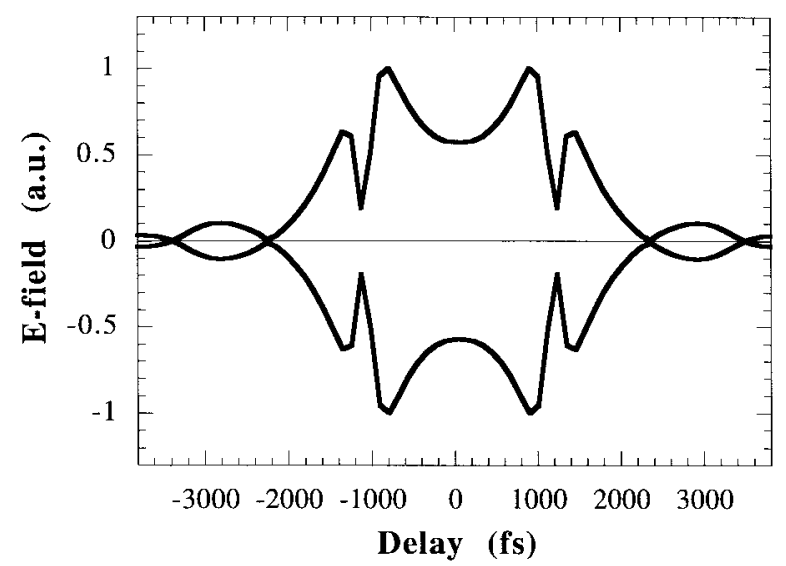

(c)

Fig. 4. (a) Spectrum and (b) the electric-field cross-correlation data and the extracted envelope of the diffracted temporal image when the Fourier transform of a single slit is projected onto the PRQW. The reference beam power density is lower than the maximum image-carrying beam power density. This shows edge enhancement of the converted square wave. The corresponding theoretical calculations of the spectrum and the temporal field envelope at $I_{1} / I_{2 \mathrm{Max}}=0.01$ are also given in (a) and (c).

such as Fourier transform and time reversal. These are singleimage processing operations.

Edge Enhancement of a Time-Domain Image: Using the experimental setup in Fig. 1, we can perform edge enhancement on the converted time-domain image by choosing an appropriate hologram-writing power density ratio. We know from (14) that the hologram in the PRQW depends only on the power density ratio of the two beams $I_{2} / I_{1}$. For the hologram pattern to be a faithful replica of the original electric field distribution, the reference beam power density must be higher than the most intense spot of the Fourier transform of the image. When this condition is not satisfied, the hologram amplitude saturates and even decreases at positions where the reference beam power density is weaker than the imagecarrying beam. Such saturation and decrease can serve to enhance the high-frequency optical frequency components at the expense of the strong peak at the fundamental frequency. This produces a high-pass filter which can lead to edge enhancement in the reconstructed images. Such effects were demonstrated in the space domain [36], [37] and used to detect dust and other small defects in periodic photolithographic masks [38].

Experimentally, the power density of the reference beam is reduced by two orders of magnitude relative to the image- carrying beam using neutral-density filters. As a result, the interference of the two writing beams (the hologram) washes out the primary maximum in the image-carrying beam and extracts the higher spatial frequency components of the slit-diffraction [sidelobes with lower power in Fig. 3(a)], which describe edges or other suddenly changing features. The diffracted spectrum from such a hologram is shown in Fig. 4(a), together with the theoretical calculation. The temporal shapes of the diffracted (or reconstructed) femtosecond outputs, which are the Fourier transform of the electric field spectrum, are given in Fig. 4(b), showing the enhanced edges when compared with the time-domain image in Fig. 3(b). The side wings in Fig. 4(b) are also expected from the theoretical Fourier transform of (14), which are shown in Fig. 4(c). Physically, the high-pass filtering operation in this experiment transforms the rising and falling edges of the input image into the observed derivative-like features.

Fourier Transform of a Time-Domain Image: The output time-domain image is the Fourier transform of the diffracted spectrum from the hologram. If the hologram corresponds to the space-domain image itself, then the resulting time-domain image is related to the Fourier transform of the space image. Experimentally, this is realized by directly imaging a mask (amplitude or phase) onto the PRQW in the pulse shaper. 


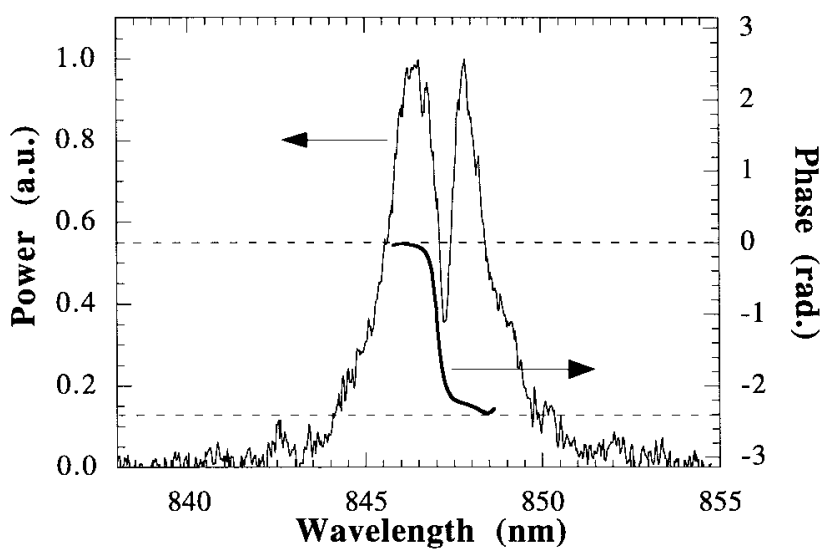

(a)

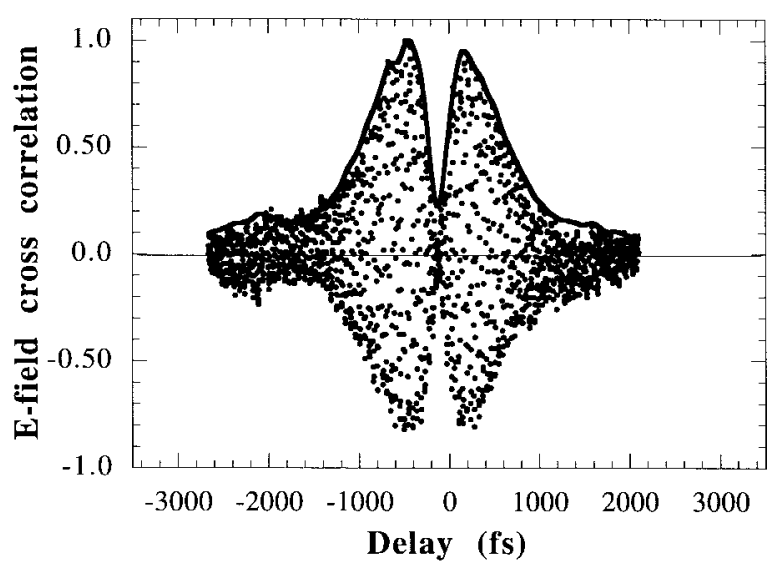

(b)

Fig. 5. (a) Spectrum and (b) electric-field cross-correlation data and the extracted envelope of the shaped femtosecond pulse when a phase step mask is imaged onto the PRQW, showing the Fourier transform of a phase-step mask (double pulse)

As the first example of this processing operation, a fixed phase mask is placed into the image-carrying beam and imaged onto the PRQW using a spherical lens with a focal length of $200 \mathrm{~mm}$. The photolithographically etched mask contains a phase step of $\pi$ for a wavelength of about $620 \mathrm{~nm}$. For our writing beams from the diode laser at around $685-690 \mathrm{~nm}$, the phase step $\sigma$ has a value of $0.9 \pi$ or smaller, depending on the incident angle of the writing beam. This phase step changes the phase of one half of the writing beam by the amount of $\sigma$ with respect to the other half, giving rise to a $\sigma$ phase step in the hologram. The diffracted beam will experience a phase function

$$
H(\omega)= \begin{cases}1, & -\infty<\omega \leq \omega_{c} \\ \exp (i \sigma) & \omega_{c} \leq \omega<\infty\end{cases}
$$

with the center frequency $\omega_{c}$. The output electric field of (1) becomes

$$
E_{D}(\omega)=E_{\mathrm{IN}}(\omega) F_{T}(\omega) F_{D}(\omega) H(\omega) .
$$

The experimental spectrum of the diffracted pulse is shown in Fig. 5(a). By interfering this spectrum with the spectrum of the reference pulse having a chosen time delay (spectral interferometry), the phase step in the femtosecond output can be extracted, which is also shown in the figure. The measured

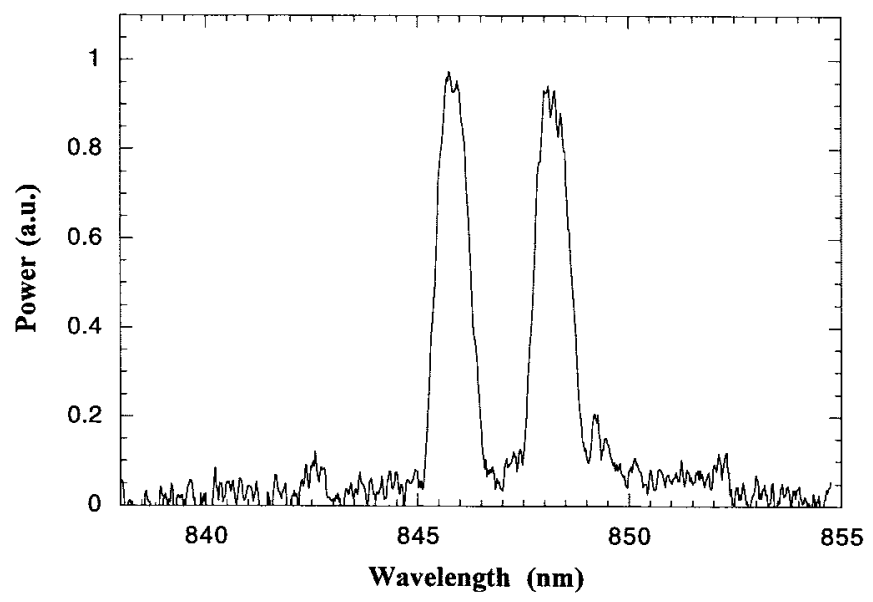

(a)

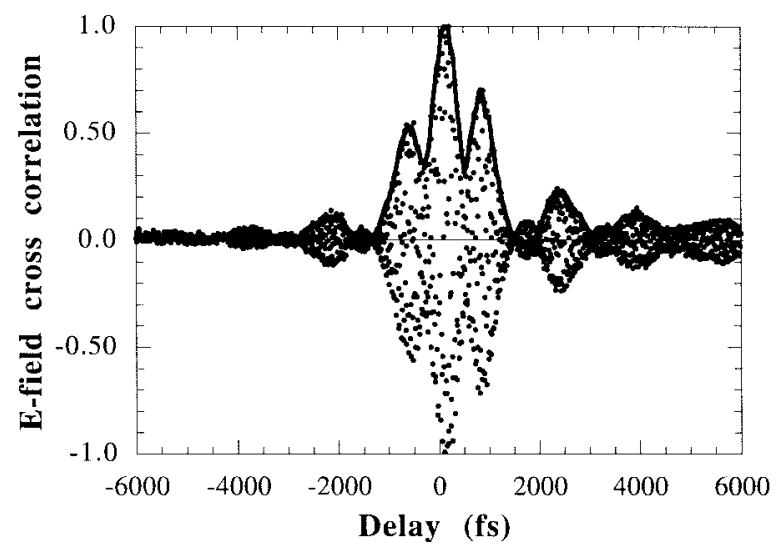

(b)

Fig. 6. (a) Spectrum and (b) electric-field cross-correlation data and the extracted envelope of the shaped femtosecond pulse when a double-slit amplitude mask is imaged onto the PRQW, showing the Fourier transform of the double-slit.

phase step is $0.8 \pi$, which is close to the estimated value. The notch in the spectrum is due to the destructive interference of the frequency components focused near the mask step [39]. The output pulse in the time domain is the Fourier transform of $E_{D}(\omega)$ in (17), which results in a double pulse. The experimental data of the electric-field cross correlation and the envelope extracted from the data are shown in Fig. 5(b).

In Fig. 6, we show a second example, in which a double-slit amplitude mask is imaged onto the PRQW with an magnification of 2.2. The magnification was chosen for technical simplicity. An identical lens $(f=200 \mathrm{~mm})$ is introduced in the reference beam to compensate the wavefront difference in the two writing beams due to the magnification. The lens in the reference beam also compensates the quadratic phase shift caused by the single imaging lens (although it is not severe in our experiments). An alternative would be using the canonical $1 f-2 f-1 f$ imaging system instead of the single lens in the imaging beam, which in fact images the wavefronts with a magnification restricted to -1 . The slit width is approximately $250 \mu \mathrm{m}$ with a separation of $300 \mu \mathrm{m}$. The double slit pattern at the Fourier plane allows only two discrete frequency groups to be diffracted [Fig. 6(a)], resulting in a double-slit interference pattern in the time domain. In this case, the function $H(\omega)$ 
in (17) is

$$
H(\omega)=B\left(\omega-\omega_{s} / 2\right)+B\left(\omega+\omega_{s} / 2\right)
$$

with the frequency separation $\omega_{s}$ and the box function of width $\omega_{w}:$

$$
B(\omega)=\left\{\begin{array}{ll}
1 & |\omega| \leq \omega_{w} / 2 \\
0 & |\omega|>\omega_{w} / 2
\end{array} .\right.
$$

The Fourier transform of (17) using $H(\omega)$ in (18) gives a typical double-slit interference pattern, as we observed experimentally [Fig. 6(b)], which is the Fourier transform of the double-slit.

Using Fourier filtering, other processing operations for single time-domain images such as noise suppression and time reversal can also be realized. For example, time reversal can be obtained by using an inverting telescope in the image-carrying beam in the hologram writing geometry.

\section{B. Processing of Multiple Images: Correlation of Time-Domain Images}

In optical processing, one of the most ubiquitous operations is the correlation, which is essentially a method for assessing the degree of similarity between two images. For two 1-D images $f(x)$ and $g(x)$, the correlation is defined as

$$
f(x) \odot g(x)=\int_{-\infty}^{\infty} f\left(x^{\prime}\right) g\left(x+x^{\prime}\right) d x^{\prime} .
$$

The relationship between (20) and the Fourier transform of the input images, known as the correlation theorem, is useful in image processing and allows one to correlate in the time domain by simple multiplication in the frequency domain. That is, if $f(x)$ has the Fourier transform $F(\omega)$ and $g(x)$ has the Fourier transform $G(\omega)$, then the Fourier transform of the product $F(\omega) G^{*}(\omega)$ is the correlation $f(x) \odot g(x)$ :

$$
\mathcal{F}\left\{F(\omega) G^{*}(\omega)\right\}=f(x) \odot g(x)
$$

where $\mathcal{F}$ represents the Fourier transform. Experimentally, the product of the Fourier transform of the first image and the phase conjugate of the Fourier transforms of the second image are obtained by projecting the Fourier transform of a space-domain image in each of the two writing beams onto the PRQW using Fourier lenses. The interference term of the power density distribution on the PRQW $I(x)=$ $\left|E_{1}(x)+E_{2}(x)\right|^{2}$ is proportional to the product of the two fields $E_{1}(x) E_{2}^{*}(x)$, which gives the distribution or envelope of the hologram modulation as long as the power density response of the PRQW remains linear, which was proven to be true in our experiments. The field diffraction from the hologram then is related to the product of the Fourier transform of the first image and the phase conjugate of the Fourier transform of the second image. The output time-domain image (the Fourier transform of this product) is then the correlation of the two input images.

We performed the correlation experiments using two identical single-slits as input images, whose Fourier transform is a

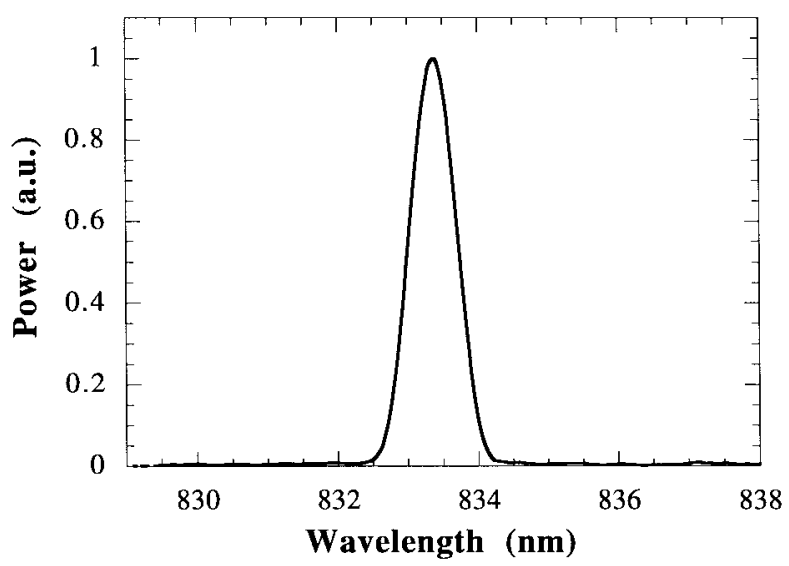

(a)

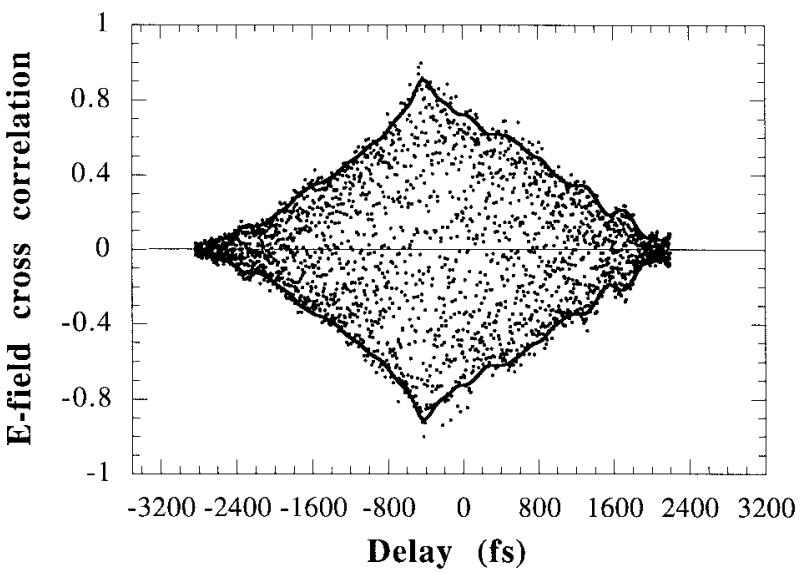

(b)

Fig. 7. (a) Spectrum and (b) electric-field cross-correlation data and the extracted envelopes of the correlation of two images (square-wave packets). The Fourier transform of the two input images are projected onto the PRQW.

sinc function as given in (15). The diffracted power spectrum is then proportional to the fourth power of the sinc function in (15), as shown in Fig. 7(a). The temporal output is the Fourier transform of this spectrum and is a triangle function, corresponding to the correlation of two square-wave packets. Fig. 7(b) shows the electric-field cross correlation of such a processed time-domain image.

Another important operation is the convolution, which is also called folding product, composition product or superposition integral. The convolution of two images $f(x), g(x)$ is given by

$$
f(x) \otimes g(x)=\int_{-\infty}^{\infty} f\left(x^{\prime}\right) g\left(x-x^{\prime}\right) d x^{\prime} .
$$

Similar to the correlation, the convolution theorem states

$$
\mathcal{F}\{F(\omega) G(\omega)\}=f(x) \otimes g(x)
$$

This is similar to (21), but with $G(\omega)$ instead of $G^{*}(\omega)$ in the product. Experimentally, the phase conjugate of $E(\omega)$ can be obtained by phase conjugating one of the hologram writing beam using a photorefractive crystal, such as $\mathrm{BaTiO}_{3}[40]$ or 
$\mathrm{KNbO}_{3}$ [41]. If $g(x)$ is an even function, then $G(\omega)$ is purely real and $G(\omega)=G^{*}(\omega)$. In this case, convolution will be the same as the correlation, as in our experiment, where the square-wave packet is a symmetric function.

\section{CONCLUSION}

We have demonstrated space-to-time conversion for generating femtosecond time-domain images which are the duplicates of the space-domain images by using PRQW's as dynamic diffractive masks in a Fourier pulse shaper. We have shown that the PRQW's are suitable for Fourier-filtering applications, and have also discussed image processing techniques for both single and multiple images. A number of processing operations, such as edge enhancement, Fourier transform, and correlation, are realized by appropriately configuring the hologram writing geometry and changing the power densities. This will allow transfer of information at a high bandwidth and bits of information to be packed together more tightly, which could potentially result in higher data rate fiber lightwave transmission systems.

\section{REFERENCES}

[1] A. M. Weiner, J. P. Heritage, and E. M. Kirschner, "High resolution femtosecond pulse shaping," J. Opt. Soc. Amer., vol. B5, pp. 1563-1572, 1988.

[2] A. M. Weiner, "Femtosecond optical pulse shaping and processing," Prog. Quantum Electron, vol. 19, pp. 161-237, 1995.

[3] M. M. Wefers, K. A. Nelson, and A. M. Weiner, "Multidimensional shaping of ultrafast optical waveforms," Opt. Lett., vol. 21, pp. 746-748, 1996.

[4] A. M. Weiner, D. E. Leaird, J. S. Patel, and J. R. Wullert, II, "Programmable femtosecond pulse shaping by using a multielement liquid crystal phase modulator," Opt. Lett., vol. 15, pp. 326-328, 1990.

[5] _ "Programmable shaping of femtosecond optical pulses by use of a 128-element liquid crystal phase modulator," IEEE J. Quantum Electron., vol. 28, pp. 908-920, 1992.

[6] M. M. Wefers and K. A. Nelson, "Programmable phase and amplitude femtosecond pulse shaping," Opt. Lett., vol. 18, pp. 2032-2034, 1993.

[7] M. M. Wefers and K. A. Nelson, "Analysis of programmable ultrashort waveform generation using liquid-crystal spatial light modulators," $J$. Opt. Soc. Amer., vol. B12, pp. 1343-1362, 1995

[8] C. Hillegas, J. X. Tull, D. Goswami, D. Strickland, and W. S. Warran, "Femtosecond laser pulse shaping by use of microsecond radiofrequency pulses," Opt. Lett., vol. 19, pp. 737-739, 1994.

[9] M. C. Nuss and R. L. Morisson, "Time-domain images," Opt. Lett., vol. 20, pp. 740-742, 1995.

[10] K. Ema, "Real-time ultrashort pulse shaping and pulse shape measurement using a dynamic grating," Jpn. J. Appl. Phys., vol. 30, pp. L2046-L2049, 1991

[11] Y. Ding, R. M. Brubaker, D. D. Nolte, M. R. Melloch, and A. M. Weiner, "Femtosecond pulse shaping by dynamic holograms in photorefractive multiple quantum wells," Opt. Lett., vol. 22, pp. 718-720, 1997.

[12] K. Ema, M. Kuwata-Gonokami, and F. Shimizu, "All-optical sub-Tbits/s serial-to-parallel conversion using excitonic giant nonlinearities," Appl. Phys. Lett., vol. 59, pp. 2799-2801, 1991.

[13] M. C. Nuss, M. Li, T. H. Chiu, A. M. Weiner, and A. Partovi, "Time-tospace mapping of femtosecond pulses," Opt. Lett., vol. 19, pp. 664-666, 1994.

[14] Y. T. Mazurenko, S. E. Putilin, A. G. Spiro, A. G. Beliaev, V. E. Yashin, and S. A. Chizhov, "Ultrafast time-to-space conversion of phase by the method of spectral nonlinear optics," Opt. Lett., vol. 21, pp. 1753-1755, 1996.

[15] P. C. Sun, Y. T. Mazurenko, W. S. C. Chang, P. K. L. Yu, and Y. Fainman, "All optical parallel-to-serial conversion by holographic spatialto-temporal frequency encoding," Opt. Lett., vol. 20, pp. 1728-1730, 1995.

[16] P. C. Sun, Y. T. Mazurenko, and Y. Fainman, "Femtosecond pulse imaging: Ultrafast optical oscilloscope," J. Opt. Soc. Amer., vol. A14, pp. 1159-1170, 1997.
[17] Y. Ding, D. D. Nolte, M. R. Melloch, and A. M. Weiner, "Realtime edge enhancement of femtosecond time-domain images by use of photorefractive quantum wells," Opt. Lett., vol. 22, pp. 1101-1103, 1997.

[18] Y. T. Mazurenko, "Holography of wavepackets," Appl. Phys., vol. B50, pp. 101-114, 1990.

[19] A. M. Weiner, D. E. Leaird, D. H. Reitze, and E. G. Paek, "Femtosecond spectral holography," IEEE J. Quantum. Electron., vol. 28, pp. 2251-2261, 1992.

[20] A. M. Weiner, D. E. Leaird, D. H. Reitze, and E. G. Paek, "Spectral holography of shaped femtosecond pulses," Opt. Lett., vol. 17, pp. 224-226, 1992.

[21] A. M. Weiner and D. E. Leaird, "Femtosecond pulse processing by second-order spectral holography," Opt. Lett., vol. 19, pp. 123-125, 1994.

[22] K. Naganuma, K. Mogi, and H. Yamada, "General method for ultrashort light pulse chirp measurement," IEEE J. Quantum Electron, vol. 25, pp. 1225-1233, 1989.

[23] K. G. Purchase, D. J. Brady, and K. Wagner, "Time-of-flight cross correlation on a detector array for ultrafast packet detection," Opt. Lett. vol. 18 , pp. 2129-2131, 1993.

[24] D. D. Nolte and M. R. Melloch, "Photorefractive quantum wells and thin films," in Photorefractive Effects and Materials, D. D. Nolte, Ed. Dordrecht, The Netherlands: Kluwer, 1995.

[25] Q. N. Wang, R. M. Brubaker, D. D. Nolte, and M. R. Melloch, "Photorefractive quantum wells: Transverse Franz-Keldysh geometry," J. Opt. Soc. Amer., vol. B9, pp. 1626-1641, 1992.

[26] R. M. Brubaker, Q. N. Wang, D. D. Nolte, E. S. Harmon, and M. R. Melloch, "Steady-state four-wave-mixing in photorefractive quantum wells with femtosecond pulses," J. Opt. Soc. Amer., vol. B11, pp. 1038-1044, 1994.

[27] R. A. Athale and K. Raj, "Fourier-plane filtering by a thick grating: A space-bandwidth analysis," Opt. Lett., vol. 17, pp. 880-882, 1992.

[28] C. De Matos, A. Le Corre, H. L'Haridon, B. Lambert, S. Salan, J. Pleumeekers, and S. Gosselin, "Photorefractive p-i-n diode quantum well operating at $1.55 \mu \mathrm{m}, "$ Appl. Phys. Lett., vol. 68, pp. 517-519, 1996.

[29] R. M. Brubaker, Y. Ding, D. D. Nolte, M. R. Melloch, and A. M. Weiner, "Bandwidth-limitted diffraction of femtosecond pulses from photorefractive quantum wells," IEEE J. Quantum. Electron., vol. 33, pp. 2150-2158, 1997.

[30] M. Cardona, "Optical constants of insulators: Dispersion relations," in Optical Properties of Solids, S. Nudelman and S. S. Mitra, Eds. New York: Plenum, 1969.

[31] L. Lepetit, G. Chriaux, and M. Joffre, "Linear techniques of phase measurement by femtosecond spectral interferometry for applications in spectroscopy," J. Opt. Soc. Amer., vol. B12, pp. 2467-2474, 1995.

[32] D. S. Chemla, D. A. B. Miller, P. W. Smith, A. C. Gossard, and W. Wiegmann, "Room temperature excitonic nonlinear absorption and refraction in GaAs/AlGaAs multiple quantum wells structures," IEEE $J$. Quantum. Electron., vol. 20, pp. 265-275, 1984.

[33] D. D. Nolte, Q. N. Wang, and M. R. Melloch, "Robust infrared gratings in photorefractive quantum wells generated by an above-band-gap laser," Appl. Phys. Lett., vol. 58, pp. 2067-2069, 1991.

[34] P. Refregier, L. Solymar, H. Rajbenbach, and J. P. Huignard, "Two-beam coupling in photorefractive $\mathrm{Bi}_{12} \mathrm{SiO}_{20}$ crystals with moving gratings: Theory and experiments," J. Appl. Phys., vol. 58, pp. 45-57, 1985.

[35] G. A. Brost, K. M. Magde, J. J. Larkin, and M. T. Harris, "Modulation dependence of the photorefractive response with moving gratings: Numerical analysis and experiment," J. Opt. Soc. Amer., vol. B11, pp. 1764-1779, 1994.

[36] J. P. Huignard and J. P. Herriau, "Real-time coherent object edge reconstruction with $\mathrm{Bi}_{12} \mathrm{SiO}_{20}$ crystals," Appl. Opt., vol. 17, pp. 2671-2672, 1978.

[37] J. Feinberg, "Real-time edge enhancement using the photorefractive effect," Opt. Lett., vol. 5, pp. 330-332, 1980.

[38] E. Ochoa, J. W. Goodman, and L. Hesselink, "Real-time enhancement of defects in a periodic mask using photorefractive $\mathrm{Bi}_{12} \mathrm{SiO}_{20}$," Opt. Lett., vol. 10, pp. 430-432, 1985.

[39] R. N. Thurston, J. P. Heritage, A. M. Weiner, and W. J. Tomlinson, "Analysis of picosecond pulse shape synthesis by spectral masking in a grating pulse compressor," IEEE J. Quantum. Electron., vol. QE-22, pp. 682-696, 1986

[40] J. Feinberg, "Self-pumped continuous-wave phase conjugator using internal reflection," Opt. Lett., vol. 7, pp. 486-488, 1982.

[41] Y. Ding, Z. G. Zhang, H. J. Eichler, D. Z. Shen, X. Y. Ma, and J. Y. Chen, "Mirrorless self-pumped phase conjugation in photorefractive $\mathrm{KNbO}_{3}: \mathrm{Mn}$ and $\mathrm{KNbO}_{3}$ :Co.," Opt. Lett., vol. 20, pp. 686-688, 1995. 


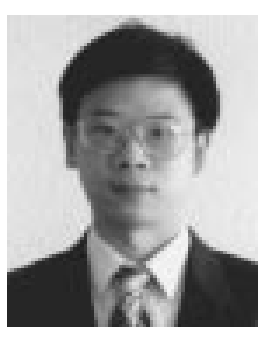

Y. Ding received the B.S. and M.S. (with honors) degrees from Tsinghua University, China, in 1985 and 1987, respectively, and the Dr. rer. nat. (Ph.D.) degree from Technical University of Berlin, Berlin, Germany, in 1992.

His thesis was on the photorefractive effects in III-V and II-VI semiconductors, where he achieved the largest two-wave mixing gain in InP:Fe at 1.06 $\mu \mathrm{m}$. From 1993 to 1996, he was a Research Scientist in the Optical Institute at the Technical University of Berlin, Berlin, Germany, and was a major contributor to several research programs supported by the Research Ministry of the Federal Republic of Germany (BMFT), such as "optical bistability," phase conjugation," "parallel switching of solid-state laser amplifiers," with research interests in photorefractive nonlinear optics, optical phase conjugation, semiconductor lasers and amplifiers. Since July 1996, he has been a Research Associate at Purdue University, West Lafayette, IN. His current research includes femtosecond time domain image processing, semiconductor MQW's, ultrafast and fiber optics. He has $\sim 80$ journal publications and conference presentations.

Dr. Ding is a member of the Optical Society of America and the New York Academy of Sciences.

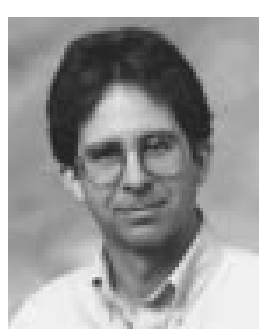

David Nolte was born in Akron, OH, on January 30, 1959. He received the bachelors degree in physics from Cornell University, Ithaca, NY, in 1981, and the Ph.D. degree in solid state physics in 1988 from the University of California at Berkeley for research leading to the first experimental measurement of bandedge hydrostatic deformation potentials in semiconductors using the concept of universal reference levels.

In September of 1988, he joined Bell Laboratories as a Post-Doctoral Member of technical staff. He joined the faculty of Physics at Purdue University, West Lafayette, IN, in 1989, where he was promoted to Associate Professor in 1994. His research interests include ultralow-power nonlinear optics, optoelectronic properties and applications of semi-insulating semiconductor heterostructures, magnetic phenomena in electrooptics and nonlinear optics, and the physics of mesoscopic point-like defects in semiconductors.

Dr. Nolte received the Presidential Young Investigator Award of the National Science Foundation, and was awarded a Research Fellowship from the Alfred P. Sloan Foundation. He has been elected a Fellow of the Optical Society of America for the demonstration and development of the science and applications of photorefractive semiconductor QW's.

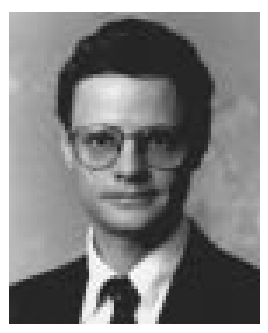

Michael R. Melloch (S'76-M'78-SM'91) received the B.S.E.E., M.S.E.E., and Ph.D. degrees from Purdue University, West Lafayette, IN, in 1975, 1976, and 1981 respectively.

From June 1976 to August 1978, he was a Design Engineer at Intel Corporation where he worked on the 8275, a CRT controller chip; the 8748, the first single-chip microcomputer; and was codesigner of the 8051, a second generation single-chip microcomputer. In February 1982, he joined the Central Research Laboratories at Texas Instruments, Inc., as a member of the Technical Staff. At Texas Instruments, his research interests centered around GaAs surface acoustic wave devices. In August 1984, he joined the School of Electrical Engineering at Purdue University as an Assistant Professor and is presently a Full Professor there and former Assistant Dean of Engineering. He has coauthored 275 conference talks, 210 technical papers, 2 book chapters, and is holder of five U.S. patents. He was involved with the first investigation of the effects of bandgap narrowing in GaAscaused by heavy impurity dopingon the electrical performance of devices and the characterization of both the electron and hole minority carrier mobilities in GaAs as a function of majority carrier concentration using a zero-field timeof-flight technique. He was involved with the development of a nonvolatile memory cell in $6 \mathrm{H}-\mathrm{SiC}$; the development of the first digital integrated circuits and CCD's in SiC; the development of the first SiC DIMOS power transistor; and the characterization and improvement of the $\mathrm{SiO}_{2} / \mathrm{SiC}$ interface. $\mathrm{He}$ was involved with the discovery of arsenic clusters in GaAs epilayers that were grown at low substrate temperatures by MBE and subsequently annealed, and the development of many electrical and optical applications of these composite materials. In 1993, he co-founded MellWood Laboratories with Prof. J. Woodall and Dr. E. Harmon. MellWood Laboratories is a supplier of custom epitaxy and high-speed photodetectors.

Dr. Melloch has served as a Symposium Co-Organizer for the 1993 Spring Meeting of the Material Research Society, the Program Chair for the 1994 North American Conference on Molecular Beam Epitaxy; Co-Organizer of the Eight International Conference on Superlattices, Microstructures, and Microdevices; and Associate Program Chair for the 1997 International Symposium on Compound Semiconductors. In 1993 he was elected to the Electronic Materials Committee of TMS, and has served as Secretary (1995-1997) and Vice-Chair (1997-1999). He has been guest editor for five journal special issues. Prof. Melloch is a Fellow of the American Physical Society, and a member the American Vacuum Society, the Minerals, Metals \& Materials Society, and the Optical Society of America.

Andrew M. Weiner (S'84-M'84-SM'91-F'95), for photograph and biography, see this issue, p. 331. 